\title{
Observation of Solid-Liquid Interfacial Reactions Controlled Bulk Phase Transition of Ni-rich Layered Cathode
}

\author{
Lianfeng Zou ${ }^{1}$, Zhenyu Liu ${ }^{2}$, Wengao Zhao ${ }^{3,4}$, Jianming Zheng ${ }^{3}$, Yong Yang ${ }^{4}$, Guofeng Wang ${ }^{2}$, Ji- \\ Guang Zhang ${ }^{3}$ and Chongmin Wang ${ }^{1}$ \\ 1. Environmental Molecular Sciences Laboratory, Pacific Northwest National Laboratory, Richland, \\ WA. \\ 2. Department of Mechanical Engineering and Materials Science, University of Pittsburgh, Pittsburgh, \\ PA. \\ 3. Energy and Environment Directorate, Pacific Northwest National Laboratory, Richland, WA. \\ 4. School of Energy Research, Xiamen University, Xiamen, Fujian, China.
}

Solid-liquid interfacial reactions are generally considered to alter the cycling performance of rechargeable batteries through the modification of surface properties, one of most representative cases is the development of the solid liquid interphase (SEI) layers, which play crucial roles on the correct operation of $\mathrm{Li}$ ion batteries [1]. However, the effects of solid-liquid interfacial reactions on the nanostructure of bulk lattice remains far from clear. X-ray diffraction (XRD) have been a routine tool to obtain the average crystallographic information, and the X-ray photoelectron spectroscopy (XPS) are widely used to acquire the chemical state information from the shallow surface layers of cathode. Nevertheless, monitoring the local lattice structure and chemistry evolution in the bulk upon cycling has been a challenge. Recently, the focused ion beam (FIB) coupling with scanning transmission electron microscopy (STEM) is employed to prepare the cross-sectional cathode samples and obtain the atomic layer by layer information from surface toward bulk in the battery community [2,3], which enables bridging the gap between the crystalline structure evolution and the batteries degradation and providing new insights into the improvement of battery performance. Using the e-transparent cross-sectional samples and HAADF-STEM, we report the observation of solid-liquid reactions induced bulk nanostructure evolution of Ni-rich cathodes.

The Ni-rich electrode was harvested from the cycled coin cells and the cross-sectional samples were prepared by FIB lift-out on the FEI Helios DualBeam microscope using ion beam (voltage range from 2-30kV). STEM observations were performed on $200 \mathrm{kV}$ JEOL JEM-ARM200CF microscope equipped with a probe spherical aberration corrector. The high-angle annular dark-field detector (HAADF) signals were collected from 68 to $280 \mathrm{mrad}$. Various facets of the primary particles were selected for the HAADF-STEM imaging to ensure the observation of intrinsic features.

Our STEM results demonstrates the typical "T" shape rock salt shape are developed upon cycling on both the exit of the $\{003\}$ surfaces and on the surfaces parallel to the $\{003\}$ channels. Fig. 1 shows the "T" shape distribution of the converted phase and indicates two growth stages for the layered to rock salt phase transition, in addition to the well-recognized thin uniform rock salt phase grown along the periphery of the primary particle, a second stage of highly anisotropic growths that traverse the Li 2D fast diffusion channels are observed. Our electrochemical results indicate such bulk growth contribute significantly to the capacity and voltage decay. Our atomistic simulations suggest that the Ni ions diffusion along the interlayer direction is favored while the intralyer diffusion is impeded as a result of the formation of rock salt/layered interface. Meanwhile the oxygen vacancies generated by solid-liquid 
interfacial reactions are found to segregate along the $\{104\}$ atomic planes and penetrate into the bulk lattice. The rock salt/layered interface and oxygen vacancies are concluded to operate in synergy to promote the bulk growth of rock salt phase in the Ni-rich layered materials.

\section{References:}

[1] P. Verma, P. Maire and P. Novák, Electrochimi. Acta 55 (2010), p. 6332.

[2] P. Yan et al, Nano. Lett 15 (2014), p. 514.

[3] P. Yan, et al, Chem. Mater 27 (2015), p. 5393.
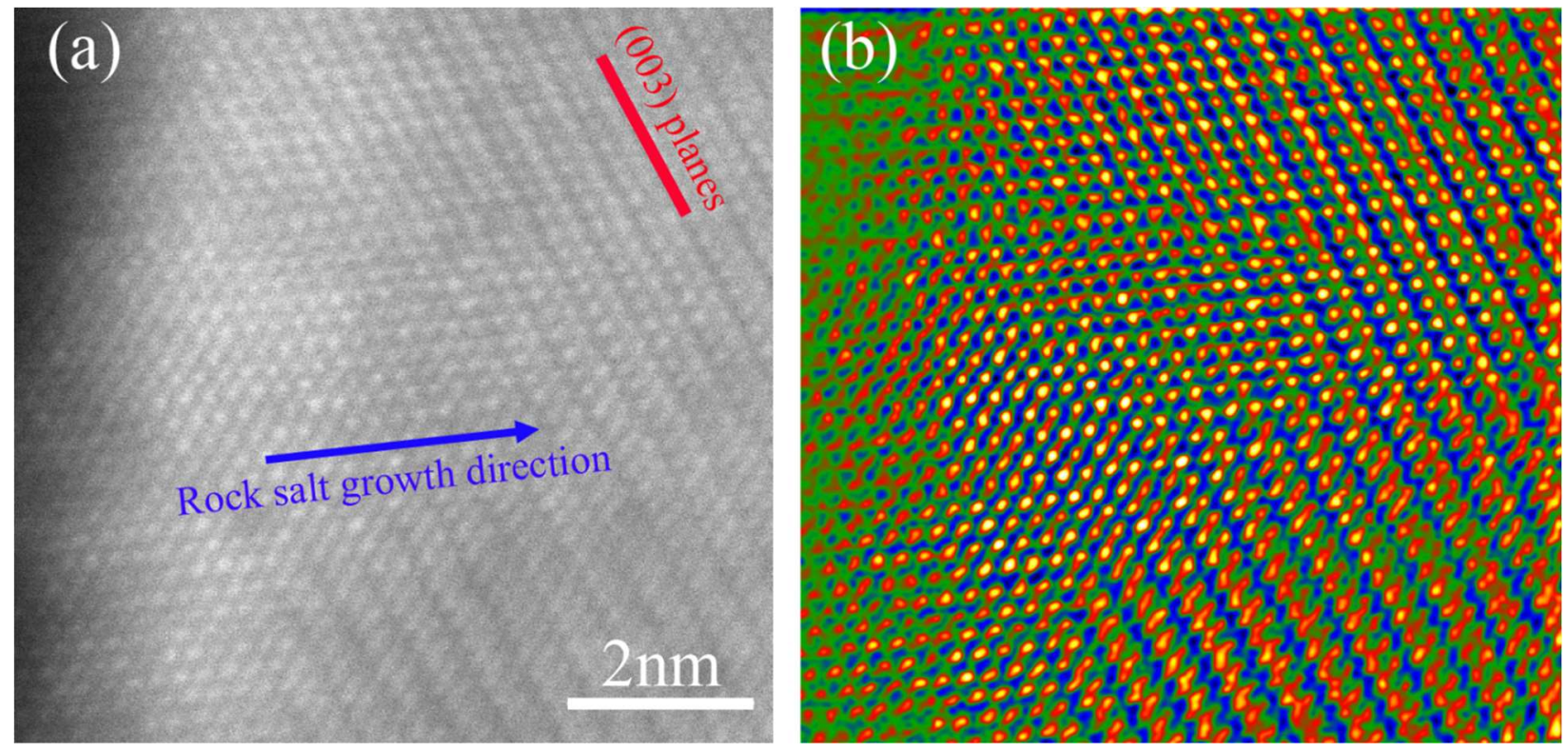

Figure 1. (a) HAADF image of rock salt bulk growth in the Ni-rich samples. (b) Intensity profile of (a). 